nanostructures, condensation des cations en solution, aspects structuraux et cinétiques de la précipitation, réactivité de surface des oxydes. La chimie de quelques éléments importants au plan technologique et environnemental, tels l'aluminium, le fer, le titane, le manganèse et le zirconium est décrite dans les trois chapitres suivants.

J'adresse de chaleureux remerciements à Philippe Belleville, Corinne Chanéac, David Chiche, Anne Duchateau, Cédric Froidefond, Julien Hernandez, Hanno Kamp, Magali Koelsh, Stéphane Lemonnier, Micaela Nazaraly, Thierry Pagès, Céline Pérégo, Agnès Pottier, David Portehault, Tamar Saison, Lionel Vayssières qui ont accompli leurs travaux de thèse sous ma (co)responsabilité. Leurs résultats ont considérablement nourri cet ouvrage.

Je remercie Élisabeth Tronc, Marc Henry, Corinne Chanéac, Sophie Cassaignon, Olivier Durupthy et tous les stagiaires post-doctorants pour les travaux que nous avons réalisés en commun. Qu'ils trouvent ici un témoignage de ma reconnaissance et de mon amitié.

J'exprime ma gratitude à tous les collègues du milieu universitaire, français et étranger, et aussi du secteur industriel qui, à travers diverses collaborations ou lors de simples échanges, ont contribué de près ou de plus loin à cet ouvrage. Je remercie tout particulièrement Benjamin Gilbert, Alain Manceau et Claudine Noguera pour leurs conseils et nos discussions passionnantes.

J'adresse enfin reconnaissance et amitié à Jacques Livage et à Clément Sanchez pour la qualité des relations scientifiques que nous avons entretenues et les heureuses années que nous avons vécues au laboratoire de Chimie de la Matière Condensée de Paris.

\title{
Liste des principales abréviations utilisées
}

EXAFS : spectroscopie d'absorption des rayons $\mathrm{X}$

MEB : microscopie électronique en balayage

MET : microscopie électronique en transmission

METHR : microscopie électronique en transmission à haute résolution $\mathrm{PCN}$ : point de charge nulle

PIE : point isoélectrique

RMN : résonance magnétique nucléaire

$\mathrm{RPE}$ : résonance paramagnétique électronique

SAXS : diffusion des rayons $\mathrm{X}$ aux petits angles 